\title{
Semi-global stabilization and output regulation of constrained linear plants via measurement feedback
}

\author{
Guoyong Shi * Ali Saberi * $\quad$ Anton A. Stoorvogel ${ }^{\dagger} \quad$ Peddapullaiah Sannuti $^{*}$
}

\section{Introduction}

Systems with state and input constraints are prevalent in the practice of control engineering. Most of the research during the past ten years focused on the systems with input constraints. The research activities during this time can broadly be divided into two directions. The first direction follows the so-called a priori design philosophy in which all the constraints are taken into account right at the onset of analysis and design. The second direction follows the so called $a$ posteriori design philosophy. In this philosophy, initially all the constraints are ignored and a satisfactory design method is used to to meet the design goals in the absence of constraints. Subsequent to this, an additional feedback layer is designed to insure stability and to reduce the loss of performance due the presence of constraints compared to the performance of the originally designed controller. Anti-windup design methodology belongs to this second category.

In addition to input constraints, state constraints also widely exist in practical control systems. Although some research activity has appeared in the literature dealing with state constraints, little attention has been paid to the structural properties associated to the constraints. Recently a new approach to state and input constraints has appeared, where the constraints on the state and/or input are modeled by a constrained output $[5,8]$. In $[5,8]$, for the first time, in the presence of state and input constraints, both stabilization and output regulation problems in a global framework as well as a semiglobal framework are formulated. Solvability conditions for such problems are developed, and whenever the solvability conditions are satisfied, explicit design methodologies to arrive at appropriate controllers or regulators are presented. The work done in $[5,8]$ focuses only on utilizing state feedback. For state feedback, and for the case of right invertible constraints, the results developed in $[5,8]$ are complete and deal with different facets of global and semi-global stabilization and output regulation. Results for non-right invertible constraints are yet to be developed.

\footnotetext{
* School of Electrical Engineering and Computer Science, Washington State University, Pullman, WA 99164-2752, U.S.A. E-mail: \{saberi, gshi\}@eecs.wsu.edu. The work of A. Saberi and G. Shi is supported by the NSF grant ECS-0000475.

$\dagger$ Department of Mathematics, and Computing Science, Eindhoven Univ. of Technology, P.O. Box 513, 5600 MB Eindhoven, The Netherlands. E-mail: A.A.Stoorvogel@tue.nl. Department of Information Technology and Systems, Delft Univ. of Technology, P.O. Box 5031, 2600 GA Delft, The Netherlands, E-mail: a.a.stoorvogel@its.tudelft.nl

$¥$ Department of Electrical and Computer Engineering, Rutgers University, 94 Brett Road, Piscataway, NJ 08854-8058, U.S.A. E-mail: sannuti@ece.rutgers.edu
}

This paper is a continuation of the work in $[5,8]$. We focus on the design of measurement feedback controllers and regulators. Some special elements needed in developing a measurement based design are discussed here. Since neither the characterization of solvability conditions nor design methodologies currently exist for global and semi-global stabilization and output regulation problems for non-right invertible constraints under state feedback, this paper considers only the case of right invertible constraints. Moreover, we only consider semi-global stabilization and output regulation problems for the reasons to be explained in the text. Due to page limitations some proofs are omitted or abbreviated. We use "im $C$ " to denote the image space of a matrix $C$, and "int $\mathcal{X}$ " to denote the interior of a set $\mathcal{X}$.

\section{Preliminaries}

In this section we describe the underlying system models and constraints, and state the problem formulations for semiglobal stabilization and semi-global output regulation. We also recall briefly the taxonomy of constraints from $[5,8]$. Consider a linear continuous-time system:

$$
\Sigma:\left\{\begin{array}{l}
\dot{x}=A x+B u \\
y=C_{y} x \\
z=C_{z} x+D_{z} u
\end{array}\right.
$$

where $x \in \mathbb{R}^{n}, u \in \mathbb{R}^{m}, y \in \mathbb{R}^{r}$ and $z \in \mathbb{R}^{p}$ are respectively the state, input, measurement output and constrained output (see Figure 1). The constrained output is subject to the constraint

$$
z(t) \in \delta, \quad \forall t \geqslant 0,
$$

where the set $\& \subset \mathbb{R}^{p}$ is a priori given and is referred to as a constraint set.

The following assumption on the constraint set $\&$ and the constrained output is used throughout the paper:

\section{Assumption 2.1}

(i) The set $\&$ is compact, convex, and contains 0 as an interior point.

(ii) $C_{z}^{T} D_{z}=0$ and $\delta=\left(\delta \cap \operatorname{im} C_{z}\right)+\left(\delta \cap \operatorname{im} D_{z}\right)$.

Given the constraint on the output, the initial state of the system must obviously be restricted. For this reason, we need to define an admissible set of initial conditions. It is straightforward to show that if the initial state does not belong to this set, then one can never avoid the constraint violation by choosing any type of feedback control law. 


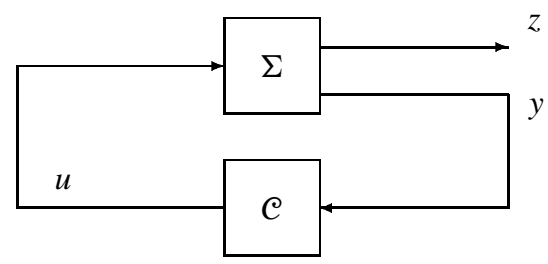

Figure 1: Closed-loop system subject to constrained output

Definition 2.2 Let the system (2.1) and a constraint set $\&$ be given. We define

$$
\mathcal{A}(\&):=\left\{x \in \mathbb{R}^{n} \mid \exists u \text { such that } C_{z} x+D_{z} u \in \delta\right\}
$$

as the admissible set of initial conditions.

Remark. In view of Assumption 2.1, we observe that the admissible set $\mathcal{A}(\delta)$ can be equivalently written as

$$
\mathcal{A}(\delta):=\left\{x \in \mathbb{R}^{n} \mid C_{z} x \in \delta\right\} .
$$

In this paper we are only concerned with constrained semiglobal stabilization and output regulation via measurement feedback. The reason that the global stabilization and output regulation problems are not discussed here is very simple. The class of systems for which these problems can be solved using measurement feedback in a global framework turns out to be very restrictive and uninteresting. In the global framework, a necessary condition for solvability is the existence of a static feedback $u=f(y)$ such that if $x(0) \in \mathcal{A}(\delta)$, then $x(t) \in \mathcal{A}(\delta)$ for all $t>0$ where

$$
\dot{x}(t)=A x(t)+B f\left(C_{y} x(t)\right) .
$$

In other words, the system must be able to satisfy the state constraints for all $t>0$ with a static output feedback. This is clearly very restrictive and therefore results for the global case are not of much interest.

We first define below the constrained semi-global stabilization of a system (2.1) via measurement feedback.

Problem 2.3 Consider a system $\Sigma$ of the form (2.1) with a constraint set $\& \subset \mathbb{R}^{p}$ satisfying Assumption 2.1. Constrained semi-global stabilization via measurement feedback is concerned with finding (if possible) a family of measurement feedbacks of the form

$$
\left\{\begin{array}{l}
\dot{v}=g(v, y, t), \quad v \in \mathbb{R}^{q} \\
u=h(v, y, t)
\end{array}\right.
$$

such that for any compact set $\mathcal{X} \subset$ int $\mathcal{A}(\&)$ and any compact set $\mathcal{V} \subset \mathbb{R}^{q}$ there exists a measurement feedback in this family such that the following conditions hold:

(i) The equilibrium point $(x, v)=(0,0)$ of the closedloop system is asymptotically stable with $\mathcal{X} \times \mathcal{V}$ contained in its region of attraction. (ii) For any $(x(0), v(0)) \in \mathcal{X} \times \mathcal{V}$, we have $z(t) \in \&$ for all $t \geqslant 0$.

For semi-global output regulation with constraints we consider the following system

$$
\Sigma_{w}:\left\{\begin{array}{l}
\dot{x}=A x+B u+E w \\
\dot{w}=S w \\
z=C_{z} x+D_{z} u \\
y=C_{y} x+D_{y} w \\
e=C_{e} x+D_{e} w
\end{array}\right.
$$

where the second equation is a model of the exosystem with state $w \in \mathbb{R}^{s}$. The initial condition $w(0)$ is assumed to be in some a priori given compact set $\mathcal{W}$. This exosystem plays a dual role. It generates an exogenous disturbance that affects the plant in terms of $E w$ which needs to be rejected but it also generates a reference signal $D_{e} w$ which we need to track. The goal of disturbance rejection and tracking is achieved by requiring that $e(t) \rightarrow 0$ as $t \rightarrow \infty$. We again impose Assumption 2.1 on the constraint for output regulation.

Problem 2.4 Consider a system of the form (2.4) with a constraint set $\& \subset \mathbb{R}^{p}$ satisfying Assumption 2.1 and a compact set $\mathcal{W} \subset \mathbb{R}^{s}$. Constrained semi-global output regulation via measurement feedback is concerned with finding (if possible) a family of measurement feedbacks of the form,

$$
\begin{aligned}
& \dot{v}=g(v, y, w, t), \quad v \in \mathbb{R}^{q} \\
& u=h(v, y, w, t)
\end{aligned}
$$

such that for any compact set $\mathcal{X} \subset$ int $\mathcal{A}(\&)$ and for any compact set $\mathcal{V} \subset \mathbb{R}^{q}$, there exists a measurement feedback in this family such that the following conditions are satisfied:

(i) In the absence of the exosystem, i.e. $w(t) \equiv 0$, the equilibrium point $(x, v)=(0,0)$ of the closed-loop system is asymptotically stable with $\mathcal{X} \times \mathcal{V}$ contained in its region of attraction.

(ii) For any $(x(0), v(0)) \in \mathcal{X} \times \mathcal{V}$ and $w(0) \in \mathcal{W}$, we have $z(t) \in \&$ for all $t \geqslant 0$ and $\lim _{t \rightarrow \infty} e(t)=0$.

(iii) For any $(x(0), v(0)) \in \mathcal{X} \times \mathcal{V}$ and $w(0) \in \mathcal{W}$, whenever we set $w(t)=0$ for $t \geqslant t_{0}$ for any $t_{0} \geqslant 0$, we have $\lim _{t \rightarrow \infty} x(t)=0, \lim _{t \rightarrow \infty} v(t)=0$, and $z(t) \in \&$ for all $t \geqslant t_{0}$.

Remark. The condition (iii) of Problem 2.4 is included to guarantee the stability of the closed-loop system if the tracking reference signal is switched off at some time $t_{0} \geq 0$. 


\subsection{Taxonomy of constraints}

Before we proceed to the statement of our main results, we recall a taxonomy of constraints as emerged from the study of constrained global and semi-global stabilization and output regulation via state feedback $[5,8]$. We denote by $\Sigma_{z u}:=$ $\left(A, B, C_{z}, D_{z}\right)$ the subsystem associated with the mapping from the input $u$ to the constrained output $z$ in system (2.1). For definitions of right invertibility and invariant zeros used in the following definition, the reader is referred to [11].

Definition 2.5 The constraints are said to be

- right invertible constraints if the subsystem $\Sigma_{z u}$ is right invertible.

- non-right invertible constraints if the subsystem $\Sigma_{z u}$ is not right invertible.

Definition 2.6 The invariant zeros of the subsystem $\Sigma_{z u}$ are called the constraint invariant zeros of system (2.1) associated with the constrained output $z$.

In the next definition we denote by $\mathbb{C}, \mathbb{C}^{-}, \mathbb{C}^{0}$, and $\mathbb{C}^{+}$respectively the set of complex numbers in the entire complex plane, open negative half complex plane, imaginary axis, and open positive half complex plane.

Definition 2.7 The constraints are said to be

- minimum phase constraints if the constraint invariant zeros of the plant are all in $\mathbb{C}^{-}$.

- weakly minimum phase constraints if the constraint invariant zeros of the plant are all in $\mathbb{C}^{-} \cup \mathbb{C}^{0}$ with at least one constraint invariant zero in $\mathbb{C}^{0}$ and those constraint invariant zeros in $\mathbb{C}^{0}$ are simple.

- weakly non-minimum phase constraints if the constraint invariant zeros of the plant are all in $\mathbb{C}^{-} \cup \mathbb{C}^{0}$ with at least one non-simple constraint invariant zero in $\mathbb{C}^{0}$.

- at most weakly non-minimum phase constraints if the constraint invariant zeros of the plant are all in $\mathbb{C}^{-} \cup$ $\mathbb{C}^{0}$.

- strongly non-minimum phase constraints if at lease one of the constraint invariant zeros of the plant is in $\mathbb{C}^{+}$.

\section{Constrained semi-global stabilization via measurement feedback}

In this section we solve the constrained semi-global stabilization via measurement feedback, as formulated in Problem 2.3.

Theorem 3.1 Consider the plant $\Sigma$ as given by (2.1) and a constraint set \& that satisfies Assumption 2.1. Assume that the constraints are right invertible. Then the constrained semi-global stabilization problem via measurement feedback as defined in Problem 2.3 is solvable if the following conditions hold:

(i) $(A, B)$ is stabilizable.

(ii) The constraints are at most weakly non-minimum phase.

(iii) The pair $\left(C_{y}, A\right)$ is observable.

Moreover, conditions (i) and (ii) are necessary.

The proof of this theorem follows by explicitly constructing a suitable observer-based measurement feedback controller. So far, there is only one linear state feedback controller available in the literature [5] by which the constrained semi-global stabilization problem is solved. For the sake of continuity of our presentation, we briefly recall from [5] the main procedure involved in the design of such a state feedback.

The design of state feedback law for a system with right invertible and at most weakly non-minimum phase constraints is based on a transformation of the original system by choosing a special coordinate basis (scb) for the subsystem $\Sigma_{z u}$ of system (2.1). More specifically, the design is accomplished in the following steps. For a system with right-invertible constraints, we can choose appropriate coordinates in the state space and the input space for the subsystem $\Sigma_{z u}$ so that the system (2.1) takes the following form [6,9],

$$
\bar{\Sigma}:\left\{\begin{aligned}
\dot{x}_{a} & =A_{a} x_{a}+K_{a} z \\
\dot{x}_{c}=A_{c} x_{c}+B_{c}\left(u_{c}+H_{a} x_{a}\right)+K_{c} z & \\
\dot{x}_{d}=A_{d} x_{d}+B_{d}\left(u_{d}+G_{a} x_{a}+G_{c} x_{c}+G_{d} x_{d}\right) & \quad+K_{d} z \\
z & =\left(\begin{array}{l}
z_{0} \\
z_{d}
\end{array}\right)=\left(\begin{array}{c}
D_{0} u_{0} \\
C_{d} x_{d}
\end{array}\right) \\
y & =C_{y a} x_{a}+C_{y c} x_{c}+C_{y d} x_{d}
\end{aligned}\right.
$$

Moreover, the eigenvalues of $A_{a}$ are the invariant zeros of the subsystem $\Sigma_{z u}$ and the matrix pair $\left(A_{c}, B_{c}\right)$ is controllable. System (3.1) can be split into two subsystems: the first one given by

$$
\Sigma_{1}:\left\{\dot{x}_{a}=A_{a} x_{a}+K_{a} z, \quad x_{a} \in \mathbb{R}^{n_{a}}\right.
$$

and the second one by

$$
\Sigma_{2}:\left\{\begin{array}{c}
\dot{x}_{c}=A_{c} x_{c}+B_{c}\left(u_{c}+H_{a} x_{a}\right)+K_{c} z, \quad x_{c} \in \mathbb{R}^{n_{c}} \\
\dot{x}_{d}=A_{d} x_{d}+B_{d}\left(u_{d}+G_{a} x_{a}+G_{c} x_{c}\right. \\
\left.\quad+G_{d} x_{d}\right)+K_{d} z, x_{d} \in \mathbb{R}^{n_{d}} \\
z=\left(\begin{array}{c}
z_{0} \\
z_{d}
\end{array}\right)=\left(\begin{array}{c}
D_{0} u_{0} \\
C_{d} x_{d}
\end{array}\right) .
\end{array}\right.
$$

By Assumption 2.1, the set $\&$ can be decomposed compatibly with the decomposition of $z$ as

$$
s=\varsigma_{0} \times \varsigma_{d}
$$

such that $z \in \S$ if and only if $z_{0} \in \wp_{0}$ and $z_{d} \in \wp_{d}$. 
The condition that the constraints are at most weakly nonminimum phase implies that all the eigenvalues of $A_{a}$ are in the closed left-half plane because $\Sigma_{1}$ represents the zero dynamics of subsystem $\Sigma_{z u}$. Hence if we view $z$ as the input to this subsystem with constraint $z(t) \in \delta$, the null controllability region of this subsystem is the entire $x_{a}$ subspace. Let $\chi$ be a compact set contained in the interior of $\mathcal{A}(\&)$ and let

$$
\begin{gathered}
\mathcal{X}_{1}=\left\{x_{a} \in \mathbb{R}^{n_{a}} \mid \exists x_{c} \in \mathbb{R}^{n_{c}}, x_{d} \in \mathbb{R}^{n_{d}}\right. \text { such that } \\
\left.\left(\begin{array}{lll}
x_{a}^{\mathrm{T}} & x_{c}^{\mathrm{T}} & x_{d}^{\mathrm{T}}
\end{array}\right)^{\mathrm{T}} \in X\right\} .
\end{gathered}
$$

We design a linear feedback $z=F_{a} x_{a}$ to stabilize this subsystem $\Sigma_{1}$. We require that, even with an exponentially decaying disturbance added to this subsystem, namely, there exist $M>0$ and $\delta>0$ such that

$$
\dot{x}_{a}=\left(A_{a}+K_{a} F_{a}\right) x_{a}+K_{a} v,
$$

with $v$ satisfying

$$
\|v(t)\| \leqslant M e^{-\delta t}
$$

for all $t \geqslant 0$ and any initial condition in the set $X$, the state trajectory is still attracted to zero while, at the same time, there exists a $\rho \in(0,1)$ such that

$$
F_{a} x_{a}(t) \in \rho \&
$$

for all $t \geqslant 0$. Such a linear feedback law can be designed via a direct method or a Riccati equation based method (see for example [4]).

However, $z$ is not a control variable. This means that we need to design $u_{0}, u_{c}, u_{d}$ for the second subsystem (3.3) such that $z$ approaches $F_{a} x_{a}$ exponentially given any initial conditions in $\mathcal{X}$. As such, we let $z=F_{a} x_{a}+v$, where $v=z-F_{a} x_{a}$. It is shown in [5] that by an appropriate design, one can guarantee that $v$ satisfies (3.5), the entire closed-loop system is asymptotically stable with $\mathcal{X}$ contained in the region of attraction, and the constraint on $z$ is maintained. The details of this part of design can be found in [5].

With this linear state feedback at hand, our goal in this section is to implement it by an observer based measurement feedback controller. However, a high-gain observer usually has a peaking phenomenon associated with it, and such a peaking in the estimate of the state is dangerous as it can potentially cause constraint violation. Hence, it should be taken care of in our design. Therefore, the design task will be completed in the following steps. First we show a lemma which states that any linear state feedback design that achieves the properties as we mentioned above is in fact robust in tolerating certain exponentially decaying disturbance of sufficiently small magnitude.

\section{Lemma 3.2 Consider the system}

$$
\Sigma_{c l}:\left\{\begin{array}{l}
\dot{x}=A_{c l} x+E_{c l} \mu \\
z=C_{c l} x+D_{c l} \mu
\end{array}\right.
$$

with $A_{c l}$ Hurwitz stable. Let $\&$ be a given set satisfying Assumption 2.1. Let $\mathcal{X} \subset$ int $\mathcal{A}(\&)$ be a compact set in $\mathbb{R}^{n}$.
Assume there exists $\rho \in(0,1)$ such for all $x(0)=x_{0} \in \mathcal{X}$ and $\mu(t)=0$, we have $z(t) \in \rho$ \& for all $t \geqslant 0$. Then there exist $\varepsilon>0, r>0$, and a compact set $\Omega(\mathcal{X}) \subset$ int $\mathcal{A}(\&)$ such that for all $x_{0} \in \mathcal{X}$ and for all $\mu(t)$ satisfying

$$
\|\mu(t)\| \leqslant \varepsilon e^{-r t}, \quad \forall t \geqslant 0
$$

we have that $z(t) \in \delta$ and $x(t) \in \Omega(\mathcal{X})$ for all $t \geqslant 0$ while $x(t) \rightarrow 0$ as $t \rightarrow \infty$.

Remark. We state this lemma for any general linear state feedback design that meets the condition stated in the theorem. Although so far there is only one linear state feedback design that has been presented in [5], this lemma subsumes all other possible linear state feedback designs that solve the constrained semi-global stabilization problem.

This lemma is the first preparatory step toward an observer design. However, if we adopt a fast observer to make an extremely accurate estimation of the state, there is an unavoidable annoying phenomenon called peaking associated with high-gain observer design. The peaking phenomenon must be taken care of seriously, for we are facing a control system with constraints. Fortunately, we have another lemma which provides us a mechanism to avoid the negative effect of peaking. As the second step, we recall the lemma here because it is instrumental to the high-gain observer design.

\section{Lemma 3.3 Consider the system}

$$
\dot{\eta}=(A-L C) \eta
$$

where $A \in \mathbb{R}^{n \times n}$ and $(C, A)$ is observable. Then, for any $N>0, \varepsilon>0, r>0$, and $\tau>0$ there exists a matrix $L$ such that $A-L C+r I$ is Hurwitz stable and

$$
\|\eta(t)\| \leqslant \varepsilon e^{-r t}
$$

for all $t \geqslant \tau$ and for all initial conditions $\eta(0) \in \mathbb{R}^{n}$ satisfying $\|\eta(0)\| \leqslant N$.

Proof : This lemma follows from the results in Izmaĭlov [2,3] and Theorem 8.2 in Sussmann and Kokotovic [10].

Proof of Theorem 3.1 : The necessity of conditions ( $i$ ) and (ii) is a consequence of the state feedback design, see [5]. The sufficiency of the conditions is proven by an explicit design as presented below.

We need a high-gain observer to estimate the state. The observer takes the standard form

$$
\dot{\hat{x}}=A \hat{x}+B u+L\left(y-C_{y} \hat{x}\right) .
$$

By the assumption that $\left(C_{y}, A\right)$ is observable, we can choose a gain matrix $L$ such that the eigenvalues of the matrix

$$
A_{\mathrm{obs}}:=A-L C_{y}
$$


can be assigned anywhere in the left-half complex plane. The estimation error $e:=\hat{x}-x$ satisfies

$$
\dot{e}=A_{\mathrm{obs}} e .
$$

Our goal is to devise a measurement feedback such that the set $\mathcal{X} \times \mathcal{V}$ is contained in the domain of attraction, meanwhile for all initial states in this set the constraints are satisfied. Due to the possible peaking of the state estimate caused by the high-gain observer, the state estimate during the short period at the beginning of time is not useful. To insure that the constraints are not violated, we saturate the control [1] so that the peaking signal does not enter the plant. The approriate level of saturation is specified below. Then, the design objective is to guarantee that the control law is functioning as closely as the state feedback law after the peaking is over. But during the short period of peaking and saturation, the state starting from $\mathcal{X}$ may drift to a larger set, say $\widehat{X}$. For this reason, we need two components in our design. One is to design from the beginning a state feedback $u=F x$ for a larger set of initial condition, say set $\widehat{x}$ which satisfies $x \subset$ int $\widehat{X} \subset$ int $\mathcal{A}(\&)$, and make sure that $\widehat{x}$ is contained in the domain of attraction and for all initial conditions in the set $\widehat{X}$ the output $z(t) \in \rho \&$ for some $\rho \in(0,1)$. One can use the design technique provided in [5] for this task. The other component is a saturation element, the level of which is specified below.

Consider the following system

$$
\dot{x}=(A+B F) x+B F e,
$$

where $e$ is the estimation error. It follows from Lemma 3.2 that if $e(t)$ satisfies

$$
\|e(t)\| \leqslant \varepsilon e^{-r t}
$$

for certain $\varepsilon \in(0,1), r>0$ and for all $t>0$, then for all initial conditions in the set $\widehat{x}$ the constraints on $z$ are satisfied, meanwhile the state trajectory remains in a compact set $\Omega(\widehat{X}) \subset$ int $\mathcal{A}(\&)$ and the state converges to zero. Hence, there exists an $M_{1}>0$ such that $\|F x\|_{\infty} \leqslant M_{1}$ for all $x(0) \in \widehat{X}$ and for all $e$ satisfying (3.12), where

$$
M_{1}=\sup _{x \in \Omega(\widehat{x})}\|F x\| .
$$

We define $M_{2}=\varepsilon\|F\|$. Let $\tau>0$ be such that

$$
\dot{x}=A x+B u
$$

satisfies $x(t) \in \widehat{X}$ for all $t \in[0, \tau]$ for all $u$ satisfying $\|u(t)\| \leqslant M_{1}+M_{2}$ and for all $x(0) \in \mathcal{X} \subset$ int $\widehat{X}$. Then, we can choose the observer gain matrix $L$ so that the error bound (3.12) holds for $t \geqslant \tau$, where $\tau, \varepsilon$, and $r$ are as specified above. Consequently, the combination of the observer and the state feedback

$$
u=\operatorname{sat}_{M_{1}+M_{2}}(F \hat{x}),
$$

where $\operatorname{sat}_{M}(\cdot)$ is a standard saturation function with saturation level $M$, has the following properties: For any given initial state $x(0) \in \mathcal{X}$, we have $x(t) \in \widehat{X}$ for all $t \in[0, \tau]$; and for $t \geqslant \tau$ we have

$$
u(t)=\operatorname{sat}_{M_{1}+M_{2}}(F \hat{x}(t))=F \hat{x}(t)=F x(t)+F e(t) .
$$

Then stabilization follows from Lemma 3.3.

\section{Constrained semi-global output reg- ulation with measurement feedback}

In this section we solve the constrained semi-global output regulation via measurement feedback as defined in Problem 2.4. As in the previous section, we focus only on the right invertible constraints in this section as well.

In the study of classical output regulation problems without constraints, it is well known that the following assumptions are standard (see [7] for details).

Assumption 4.1 There exist matrices $\Pi$ and $\Gamma$ satisfying the regulator equations,

$$
\left\{\begin{aligned}
\Pi S & =A \Pi+B \Gamma+E \\
0 & =C_{e} \Pi+D_{e} .
\end{aligned}\right.
$$

Assumption 4.2 The matrix $S$ has all its eigenvalues in the closed right-half plane.

Assumption 4.3 The pair $(A, B)$ is stabilizable.

Assumption 4.4 The pair

$$
\left.\left[\begin{array}{ll}
C_{y} & D_{y}
\end{array}\right),\left(\begin{array}{cc}
A & E \\
0 & S
\end{array}\right)\right]
$$

is observable.

Note that assumptions 4.1 and 4.3 are necessary and assumption 4.2 is natural. However, assumption 4.4 is not necessary and can be relaxed. For a detailed discussion see [7].

Under these assumptions, the solvability conditions for the constrained semi-global output regulation via measurement feedback are stated in the following theorem.

Theorem 4.5 Consider a system of the form (2.4) with a constraint set $\&$ satisfying Assumption 2.1 and a compact set $W \subset \mathbb{R}^{s}$. Assume that the constraint is right invertible and let Assumptions 4.1 to 4.4 be satisfied. Then the constrained semi-global output regulation via measurement feedback as defined in Problem 2.4 is solvable if the following conditions hold:

(i) The constraint is at most weakly non-minimum phase.

(ii) There exists a $\rho \in(0,1)$ such that for all $w(0) \in \mathcal{W}$

$$
\left(C_{z} \Pi+D_{z} \Gamma\right) w(t) \in(1-\rho) s, \text { for all } t \geqslant 0 \text {. }
$$

(iii) The signals $\Pi w$ and $\Gamma w$ are bounded. 
Moreover, condition (i) is necessary.

We need some preparation for the proof of this theorem. For the purpose of design, it would be helpful to build a connection between the output regulation problem and the stabilization problem by utilizing the regulator equations (4.1) in Assumption 4.1. Let $(\Pi, \Gamma)$ be a solution to the regulator equations (4.1). Define the following new variables:

$$
\tilde{x}=x-\Pi w, \quad \tilde{u}=u-\Gamma w, \quad \tilde{z}=z-\Lambda w,
$$

where $\Lambda=C_{z} \Pi+D_{z} \Gamma$. Then it is readily verified that system (2.4) in terms of the new variables becomes

$$
\left\{\begin{array}{l}
\dot{\tilde{x}}=A \tilde{x}+B \tilde{u} \\
\tilde{z}=C_{z} \tilde{x}+D_{z} \tilde{u} \\
e=C_{e} \tilde{x} .
\end{array}\right.
$$

Note that in (4.3) the dynamics of exosystem has been absorbed in the dynamics of the new variables. System (4.3) has a remarkable property, it tells us that the output regulation can be achieved by designing a controller that stabilizes this system. That is, if we design $\tilde{u}$ such that the closedloop system is internally stable, then we have $e(t) \rightarrow 0$ as $t \rightarrow \infty$, which is one of the goals for output regulation. However, this does not solve the whole problem, for we have constraints on the output. In other words, in addition to achieving regulation, the control law constructed must also guarantee that $z=\tilde{z}+\Lambda w$ satisfies the constraints, i.e. $\tilde{z}(t)+\Lambda w(t) \in \delta$ for all $t \geqslant 0$.

Based on the idea said above, a state feedback design for constrained output regulation has been proposed in [8]. In that design some special care has been taken to guarantee that the constraint is not violated. In order to implement such a state feedback regulation law with an observer, we need a robustness lemma similar to Lemma 3.2 for output regulation.

Lemma 4.6 Consider the system

$$
\tilde{\Sigma}_{c l}:\left\{\begin{array}{l}
\dot{\tilde{x}}=A_{c l} \tilde{x}+E_{c l} \mu \\
\tilde{z}=C_{c l} \tilde{x}+D_{c l} \mu
\end{array}\right.
$$

where $\tilde{x}$ and $\tilde{z}$ are as defined in (4.2), and $A_{c l}$ is a Hurwitz stable matrix. Let 8 be a given set satisfying Assumption 2.1. Let $\mathcal{X} \subset$ int $\mathcal{A}(\&)$ be a compact set in $\mathbb{R}^{n}$ and $\mathcal{W}$ a compact set in $\mathbb{R}^{s}$. Let the condition (ii) in Theorem 4.5 hold. Assume there exists $\rho_{1} \in(\rho, 1)$ such for all $x(0) \in \mathcal{X}, w(0) \in \mathcal{W}$, and $\mu(t)=0$, we have $z(t) \in \rho_{1} \&$ for all $t \geqslant 0$. Then there exist $\varepsilon>0, r>0$, and a compact set $\Omega(\mathcal{X}) \subset$ int $\mathcal{A}(\&)$ such that for all $x_{0} \in \mathcal{X}$ and for all $\mu(t)$ satisfying

$$
\|\mu(t)\| \leqslant \varepsilon e^{-r t}, \quad \forall t \geqslant 0
$$

we have that $z(t) \in S$ and $x(t) \in \Omega(X)$ for all $t \geqslant 0$, and $x(t) \rightarrow 0$ as $t \rightarrow \infty$.

Proof of Theorem 4.5 : The proof follows an idea similar to that in the proof of Theorem 3.1. We omit the details due to the space limitation.

\section{Conclusion}

In this paper we developed the design of observer-based measurement feedback controllers for constrained semi-global stabilization and constrained semi-global output regulation for linear systems with right invertible constraints. The observers use high-gain feedback. To avoid the possible constraint violation caused by peaking phenomenon associated with an high-gain observer, appropriate saturation mechanisms are employed in the control laws. It turns out that the solvability conditions for the two problems under the measurement feedback are similar to those developed in $[5,8]$ where only state feedback laws were considered.

A major open problem is the case of non-right invertible constraints which remains a challenging and difficult problem.

\section{References}

[1] F. Esfandiari and H.K. Khalil. Output feedback stabilization of fully linearizable systems. Int. J. Contr., 56(5):1007-1037, 1992.

[2] R.N. Izmaŭlov. "peak" effect in stationary linear systems in scalar inputs and outputs. Automation and Remote Control, 48(8, part 1):1018-1024, 1987. Translated from Avtomatika i Telemekhanika, 1987 , No. 8, pp. 56-62.

[3] R.N. Izmaĭlov. The peak effect in stationary linear systems with multivariate inputs and outputs. Automation and Remote Control, 49(1, part 1):40-47, 1988. Translated from Avtomatika i Telemekhanika, 1988, No. 1, pp. 52-60.

[4] Z. Lin, A.A. Stoorvogel, and A. Saberi. Output regulation for linear systems subject to input saturation. Automatica, 32(1):29-47, 1996.

[5] A. Saberi, J. Han, and A.A. Stoorvogel. Constrained stabilization problems for linear plants. Automatica, 38(4):639-654, 2002.

[6] A. Saberi and P. Sannuti. Squaring down of non-strictly proper systems. Int. J. Contr., 51(3):621-629, 1990.

[7] A. Saberi, A.A. Stoorvogel, and P. Sannuti. Control of linear systems with regulation and input constraints. Communication and Control Engineering Series. Springer Verlag, 2000.

[8] A. Saberi, A.A. Stoorvogel, G. Shi, and P. Sannuti. Output regulation of linear plants subject to constraints. Submitted for publication, 2001.

[9] P. Sannuti and A. Saberi. Special coordinate basis for multivariable linear systems - finite and infinite zero structure, squaring down and decoupling. Int. J. Contr., 45(5):16551704, 1987.

[10] H.J. Sussmann and P.V. Kokotovic. The peaking phenomenon and the global stabilization of nonlinear systems. IEEE Trans. Aut. Contr., 36(4):424-440, 1991.

[11] H.L. Trentelman, A.A. Stoorvogel, and M.L.J. Hautus. Linear multivariable systems. Communication and Control Engineering Series. Springer Verlag, 2001. 